\title{
X-ray polarization: General formalism and polarization analysis
}

\author{
C. Detlefs ${ }^{1, a}$, M. Sanchez del Rio ${ }^{1}$, and C. Mazzoli ${ }^{2}$ \\ 1 European Synchrotron Radiation Facility, BP. 220, 6 rue Jules Horowitz \\ 38043 Grenoble Cedex, France \\ 2 Politecnico di Milano, Dipartimento di Fisica, Piazza Leonardo da Vinci 32, 20133 Milano, \\ Italy
}

Received 23 October 2011 / Received in final form 23 March 2012

Published online 15 June 2012

\begin{abstract}
The polarization of X-rays plays an outstanding role in experimental techniques such as non-resonant magnetic X-ray scattering and resonant X-ray scattering of magnetic and multipolar order. Different instrumental methods applied to synchrotron light can transform its natural polarization into an arbitrary polarization state. Several synchrotron applications, in particular in the field of magnetic and resonant scattering rely on the improvement in the signal/noise ratio or the deeper insight into the ordered state and the scattering process made possible through these polarization techniques. Here, we present the mathematical framework for the description of fully and partially polarized X-rays, with some applications such as linear X-ray polarization analysis for the determination of the scattered beam's polarization, and the Ge K-edge resonant scattering.
\end{abstract}

\section{Mathematical description of polarized light}

Previous reviews of X-ray polarization phenomena are mostly focused on aspects related to crystal dynamical diffraction [1,2]. Several publications discuss the polarization dependent scattering amplitudes for non-resonant magnetic $[3,4]$ and magnetic and higher order multipolar resonant scattering [5], without introducing the formalism. Theoretical work on the polarization dependence of the resonant cross section mostly uses a tensor notation that is beyond the scope of this paper [6-9]. We here present a description of X-ray polarization based on classical electrodynamics with a view towards applications in magnetic scattering research with synchrotron radiation.

$\mathrm{X}$-rays are transverse electro-magnetic waves [10], just like visible light, so that the description of polarized optics in the visible regime may be applied $[11,12]$. The polarization state of an isolated wave with wave vector $\boldsymbol{k}$ and photon energy $\hbar \omega$,

$$
\boldsymbol{E}(t, \boldsymbol{r})=\Re e\left[\left(V_{1} \hat{\boldsymbol{\epsilon}}_{1}+V_{2} \hat{\boldsymbol{\epsilon}}_{2}\right) \cdot e^{-i(\omega t-\boldsymbol{k} \cdot \boldsymbol{r}}\right]
$$

\footnotetext{
${ }^{a}$ e-mail: detlefs@esrf.fr
} 


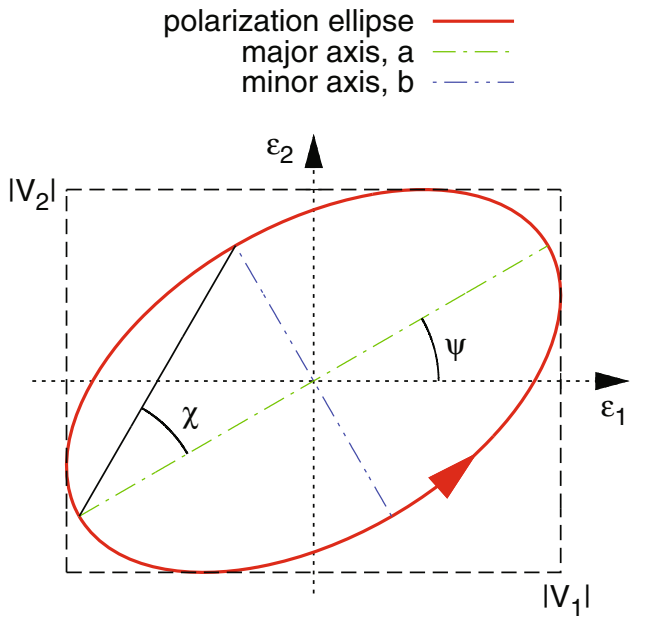

Fig. 1. The polarization ellipse of a general (elliptically polarized) beam. In this example $\psi=\chi=30^{\circ}$ (Eqs. (6) and (7), yielding $P_{1}=1 / 4, P_{2}=\sqrt{3} / 4$ and $P_{3}=\sqrt{3} / 2$. For lefthanded polarization $\left(P_{3}>0\right)$, the electric field vector rotates counterclockwise when looking into the source.

is completely defined by the components of the Jones vector, $\boldsymbol{V}=\left(\begin{array}{c}V_{1} \\ V_{2}\end{array}\right)$. The components $V_{1}$ and $V_{2}$ may be complex, e.g., in the case of circular polarization. The coordinate system $\hat{\boldsymbol{\epsilon}}_{1,2,3}$ is chosen such that $\hat{\boldsymbol{k}}=\hat{\boldsymbol{\epsilon}}_{3}, \hat{\boldsymbol{\epsilon}}_{1} \times \hat{\boldsymbol{\epsilon}}_{2}=\hat{\boldsymbol{\epsilon}}_{3}$ and $\hat{\boldsymbol{\epsilon}}_{2} \times \hat{\boldsymbol{\epsilon}}_{3}=\hat{\boldsymbol{\epsilon}}_{1}$.

For the purpose of this paper we define the intensity of the beam as $I=\left|V_{1}\right|^{2}+$ $\left|V_{2}\right|^{2}$, dropping the usual proportionality factors.

\subsection{Polarization ellipse}

Following [12], we write Eq. (1) as

$$
\frac{E_{i}(\tau)}{\left|V_{i}\right|}=\cos (\tau) \cos \left(\phi_{i}\right)+\sin (\tau) \sin \left(\phi_{i}\right)
$$

where $i=1,2 . \tau=\omega t-\boldsymbol{k} \cdot \boldsymbol{r}$ is called the propagator, $\left|V_{i}\right|$ are the maximum amplitudes, and $\phi_{i}$ the phases of the two polarizations, such that $V_{i}=\left|V_{i}\right| \exp \left(i \phi_{i}\right)$. Eliminating the explicit dependence on the propagator we obtain [12]:

$$
\frac{E_{1}^{2}(\tau)}{\left|V_{1}\right|^{2}}+\frac{E_{2}^{2}(\tau)}{\left|V_{2}\right|^{2}}-2 \frac{E_{1}(\tau) E_{2}(\tau)}{\left|V_{1}\right|\left|V_{2}\right|} \cos (\Delta \phi)=\sin ^{2}(\Delta \phi),
$$

where $\Delta \phi=\phi_{2}-\phi_{1}$ is the relative phase between the two components of the polarized beam. We see that the projection of all instantaneous electric field vectors onto a plane perpendicular to the direction of propagation $\hat{\boldsymbol{k}}$ fall onto an ellipse, the polarization ellipse [12] as shown in Fig. 1.

The major $(2 a)$ and minor $(2 b)$ axes of the ellipse are given by the equations [12]

$$
\begin{aligned}
a^{2}+b^{2} & =\left|V_{1}\right|^{2}+\left|V_{2}\right|^{2} \\
a b & =\left|V_{1}\right|\left|V_{2}\right||\sin (\Delta \phi)|,
\end{aligned}
$$


and the angle $\psi$ between the major axis and coordinate axis $\hat{\boldsymbol{\epsilon}}_{1}$ is given by

$$
\tan (2 \psi)=2 \frac{\left|V_{1}\right|\left|V_{2}\right|}{\left|V_{1}\right|^{2}-\left|V_{2}\right|^{2}} \cos (\Delta \phi)
$$

with $0 \leq \psi \leq \pi$. Finally, the angle of ellipticity $\chi$ is defined by

$$
\sin (2 \chi)=2 \frac{\left|V_{1}\right|\left|V_{2}\right|}{\left|V_{1}\right|^{2}+\left|V_{2}\right|^{2}} \sin (\Delta \phi),
$$

with $-\frac{\pi}{4} \leq \chi \leq \frac{\pi}{4}$ so that $\tan (|\chi|)=b / a$ (see Fig. 1).

\subsection{Degenerate cases of the polarization ellipse and special polarizations}

A beam with vanishing minor axis, $b=0$, is called linearly polarized. In this case the two polarizations oscillate in phase, $\Delta \phi=0$, or in antiphase, $\Delta \phi=\pi$. The angle of ellipticity is $\chi=0$, and the corresponding polarization ellipse collapses to a line.

A beam with $\sin (2 \chi)= \pm 1$, has $\sin (\Delta \phi)= \pm 1$, i.e. $\Delta \phi= \pm \pi / 2$, and $\left|V_{1}\right|=\left|V_{2}\right|$ and therefore $a=b$. In this case the angle $\psi$ is undefined. The corresponding polarization ellipse is a circle, and the beam has left- $(\Delta \phi=\pi / 2)$ or right-handed $(\Delta \phi=-\pi / 2)$ circular polarization, with electric field vectors rotating counterclockwise and clockwise, respectively, when looking into the source ${ }^{1}$.

\subsection{Poincaré-Stokes parameters}

The Poincaré-Stokes parameters $P_{1}, P_{2}$, and $P_{3}$ completely describe the state of polarization of a beam. They are defined as

$$
\begin{gathered}
P_{1}=\frac{\left|V_{1}\right|^{2}-\left|V_{2}\right|^{2}}{\left|V_{1}\right|^{2}+\left|V_{2}\right|^{2}} \\
P_{2}=\frac{\left|V_{1}+V_{2}\right|^{2}-\left|V_{1}-V_{2}\right|^{2}}{2\left(\left|V_{1}\right|^{2}+\left|V_{2}\right|^{2}\right)} \\
P_{3}=\frac{\left|V_{1}-i V_{2}\right|^{2}-\left|V_{1}+i V_{2}\right|^{2}}{2\left(\left|V_{1}\right|^{2}+\left|V_{2}\right|^{2}\right)} .
\end{gathered}
$$

$P_{1}$ and $P_{2}$ describe the state of linear polarization, and $P_{3}$ (sometimes called $P_{\mathrm{c}}$ ) the degree of circular polarization [11-14], with $P_{3}=+1$ for left-handed circular polarization. In addition, we define the degree of linear polarization as $P_{\text {lin }}=\sqrt{P_{1}^{2}+P_{2}^{2}}$. $\mathbf{P}=\left(P_{1}, P_{2}, P_{3}\right)$ is called the Poincaré-Stokes polarization vector, although it does not have the transformation properties of a vector $\left(P_{1,2}\right.$ have even parity, while $P_{3}$ has odd parity. $P_{1,2,3}$ are even under time reversal [15]). It is related to the Stokes vector, $\boldsymbol{S}$, more commonly used in optics via $S_{0}=I, S_{1,2,3}=I P_{1,2,3} \quad[11,12]$.

\footnotetext{
1 In the literature, the nomenclature for left- or right-handedness and for positive or negative helicity is not always consistent. Great care must be taken to avoid mistakes, in particular when comparing one's results with those of other groups. We derive the sign of the helicity from Eq. (16). An example for a left-handed circular wave with $P_{3}=+1$ is $V_{1}=1 / \sqrt{2}$ and $V_{2}=i / \sqrt{2}$. The phase and amplitude of this vector may be changed to obtain other waves with left-handed circular polarization.
} 
An isolated photon is $100 \%$ polarized with $P=\sqrt{P_{1}^{2}+P_{2}^{2}+P_{3}^{2}}=1$. The Poincaré-Stokes parameters are related to the polarization ellipse, Eqs. (6) and (7), as follows (see Fig. 1):

$$
\begin{gathered}
P_{1}=\cos (2 \psi) \cos (2 \chi) \\
P_{2}=\sin (2 \psi) \cos (2 \chi) \\
P_{3}=\sin (2 \chi) .
\end{gathered}
$$

\subsection{Coherency matrix}

An X-ray beam that is composed of an ensemble of independent waves may be partially polarized, i.e. $P<1$. Such a beam cannot be described by a simple Jones vector. Instead, its polarization state can be described by a density matrix $[4,14,16-18]$, in analogy to the coherency matrix used in classical optics $[11,12]$.

$$
\boldsymbol{\rho}=\left\langle\boldsymbol{V} \boldsymbol{V}^{\dagger}\right\rangle=\left(\begin{array}{c}
\left\langle V_{1} V_{1}^{\dagger}\right\rangle\left\langle V_{1} V_{2}^{\dagger}\right\rangle \\
\left\langle V_{2} V_{1}^{\dagger}\right\rangle\left\langle V_{2} V_{2}^{\dagger}\right\rangle
\end{array}\right)=\frac{I}{2}(\mathbf{1}+\boldsymbol{\sigma} \cdot \mathbf{P}),
$$

where the average, $\langle\ldots\rangle$, is taken over the ensemble of waves constituting the beam [11]. Depending on the experiment the average may require integrals over linear (beam size) and angular space (divergence), energy (bandwidth) and time (fluctuations), as the polarization may depend on each one of these parameters. $\boldsymbol{\sigma}=\left(\boldsymbol{\sigma}_{1}, \boldsymbol{\sigma}_{2}, \boldsymbol{\sigma}_{3}\right)$ represents the Pauli matrices, $\boldsymbol{\sigma}_{1}=\left(\begin{array}{cc}1 & 0 \\ 0 & -1\end{array}\right), \boldsymbol{\sigma}_{2}=\left(\begin{array}{ll}0 & 1 \\ 1 & 0\end{array}\right)$, and $\boldsymbol{\sigma}_{3}=\left(\begin{array}{cc}0 & i \\ -i & 0\end{array}\right)$.

The intensity and Poincaré-Stokes parameters for the X-ray beam above are easily extracted from the coherency matrix,

$$
\begin{aligned}
I & =\operatorname{tr}(\boldsymbol{\rho}) \\
P_{i} & =\frac{1}{I} \operatorname{tr}\left(\boldsymbol{\sigma}_{i} \cdot \boldsymbol{\rho}\right), i=1,2,3 .
\end{aligned}
$$

\subsection{Choice of coordinate system}

For an isolated wave, the choice of the coordinate axes $\hat{\boldsymbol{\epsilon}}_{1,2}$ is arbitrary $-\hat{\boldsymbol{\epsilon}}_{3}$ is given by the beam direction. When describing a scattering process these axes are usually chosen to be perpendicular $(\sigma)$ and parallel $(\pi)$ to the scattering plane. Following [4] and [5], we define the coordinate system $\hat{\boldsymbol{u}}_{1,2,3}$ through the incident and scattered beam directions, $\hat{\boldsymbol{k}}$ and $\hat{\boldsymbol{k}}^{\prime}$ (see Fig. 2).

$$
\begin{aligned}
& \hat{\boldsymbol{u}}_{1}=\frac{1}{2 \cos (\theta)}\left(\hat{\boldsymbol{k}}+\hat{\boldsymbol{k}}^{\prime}\right) \\
& \hat{\boldsymbol{u}}_{2}=\frac{1}{\sin (2 \theta)}\left(\hat{\boldsymbol{k}} \times \hat{\boldsymbol{k}}^{\prime}\right) \\
& \hat{\boldsymbol{u}}_{3}=\frac{1}{2 \sin (\theta)}\left(\hat{\boldsymbol{k}}-\hat{\boldsymbol{k}}^{\prime}\right),
\end{aligned}
$$




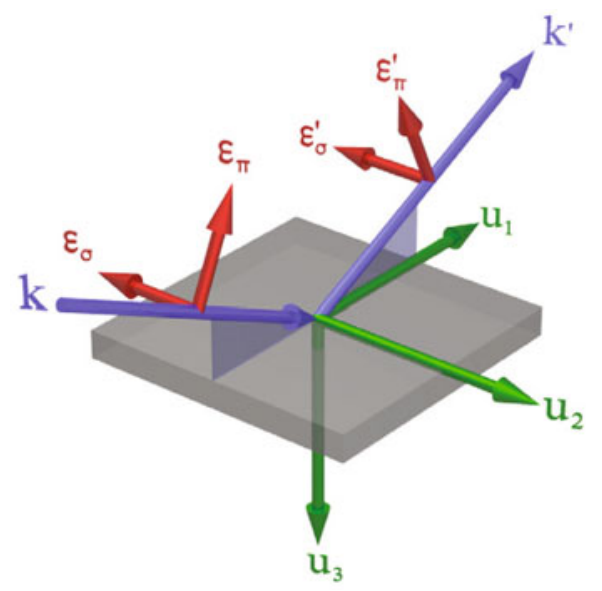

Fig. 2. Coordinate system and polarization vectors relative to the incident $(\boldsymbol{k})$ and scattered $\left(\boldsymbol{k}^{\prime}\right)$ beams.

where $2 \theta$ is the scattering angle $\left(\cos (2 \theta)=\hat{\boldsymbol{k}} \cdot \hat{\boldsymbol{k}}^{\prime}\right)$. We choose the polarization vectors as

$$
\begin{aligned}
& \hat{\boldsymbol{\epsilon}}_{\sigma}=\hat{\boldsymbol{\epsilon}}_{1}=-\hat{\boldsymbol{u}}_{2} \\
& \hat{\boldsymbol{\epsilon}}_{\pi}=\hat{\boldsymbol{\epsilon}}_{2}=\sin (\theta) \hat{\boldsymbol{u}}_{1}-\cos (\theta) \hat{\boldsymbol{u}}_{3} \\
& \hat{\boldsymbol{\epsilon}}_{\sigma}^{\prime}=\hat{\boldsymbol{\epsilon}}_{1}^{\prime}=-\hat{\boldsymbol{u}}_{2} \\
& \hat{\boldsymbol{\epsilon}}_{\pi}^{\prime}=\hat{\boldsymbol{\epsilon}}_{2}^{\prime}=-\sin (\theta) \hat{\boldsymbol{u}}_{1}-\cos (\theta) \hat{\boldsymbol{u}}_{3} .
\end{aligned}
$$

$\hat{\boldsymbol{\epsilon}}_{1}$ and $\hat{\boldsymbol{\epsilon}}_{1}^{\prime}$ are perpendicular to the scattering plane ( $\sigma$ polarization), while $\hat{\boldsymbol{\epsilon}}_{2}$ and $\hat{\boldsymbol{\epsilon}}_{2}^{\prime}$ lie within the scattering plane ( $\pi$ polarization). In the following the indices 1 and 2 may be replaced by $\sigma$ and $\pi$, respectively, when the discussion is restricted to a single scattering process with a well defined scattering plane.

In magnetic scattering one frequently encounters subsequent diffraction processes with different diffraction planes, such as vertical diffraction in the monochromator, horizontal diffraction from the sample, and vertical diffraction in a polarization analyzer. This change of reference system is accomplished through an orthogonal trans-

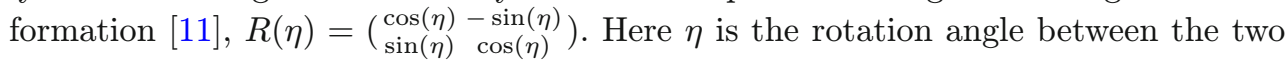
reference systems $A$ and $B$, such $\boldsymbol{E}_{B}(\tau)=R(\eta) \cdot \boldsymbol{E}_{A}(\tau)$, therefore $\boldsymbol{V}_{B}=R(\eta) \cdot \boldsymbol{V}_{A}$ and $\left(\hat{\boldsymbol{\epsilon}}_{1, B}, \hat{\boldsymbol{\epsilon}}_{2, B}\right)=\left(\hat{\boldsymbol{\epsilon}}_{1, A}, \hat{\boldsymbol{\epsilon}}_{2, A}\right) \cdot R^{-1}(\eta)$, see Fig. 3 . The density matrix transforms as $\boldsymbol{\rho}_{B}=R(\eta) \cdot \boldsymbol{\rho}_{A} \cdot R^{-1}(\eta)$, yielding the Poincaré-Stokes parameters

$$
\begin{gathered}
P_{1, B}=\cos (2 \eta) P_{1, A}+\sin (2 \eta) P_{2, A} \\
P_{2, B}=-\sin (2 \eta) P_{1, A}+\cos (2 \eta) P_{2, A} .
\end{gathered}
$$

The degree of linear polarization, $P_{\text {lin }}$, is invariant under this transformation. Also invariant are the intensity, $I$, the circular polarization, $P_{3}$ and the total degree of polarization, $P$.

$P_{1, B}$ is maximized and $P_{2, B}=0$ when the major axis of the polarization ellipse is rotated onto the $\hat{\boldsymbol{\epsilon}}_{1}$ axis, i.e. for $\sin (2 \eta)=P_{2, A} / P_{\text {lin }}$ and $\cos (2 \eta)=P_{1, A} / P_{\text {lin }}$, see Eq. (6) and Fig. 1. 


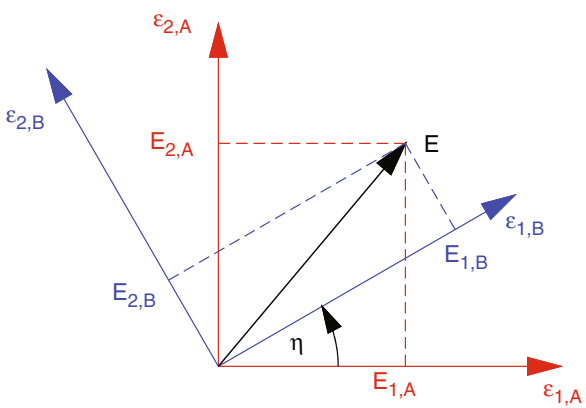

(a)



(b)

Fig. 3. A change of the coordinate system from $A$ to $B$ is effected by an orthogonal rotation matrix. (a) Transformation of the coordinate system relative to a fixed laboratory reference. (b) Transformation of the vector components in these coordinate systems.

\subsection{Jones matrices}

Jones matrices are used to describe the effect of an optical element on the beam. This formalism can be used to describe diffracting elements, filters and dichroic samples.

Let the operator $\mathbf{M}$ describe the (forward) scattering of an X-ray optical element, such as the phase plate, a sample, a polarization analyzer, or any other optical element in the path of the beam. The effect of $\mathbf{M}$ on the beam is completely described by the matrix elements of the basis vectors of polarization. They may be conveniently written in form of a $2 \times 2$ matrix, the Jones matrix [19-21],

$$
\mathbf{M}=\left(\begin{array}{c}
\left\langle\hat{\boldsymbol{\epsilon}}_{\sigma}^{\prime}|M| \hat{\boldsymbol{\epsilon}}_{\sigma}\right\rangle\left\langle\hat{\boldsymbol{\epsilon}}_{\sigma}^{\prime}|M| \hat{\boldsymbol{\epsilon}}_{\pi}\right\rangle \\
\left\langle\hat{\boldsymbol{\epsilon}}_{\pi}^{\prime}|M| \hat{\boldsymbol{\epsilon}}_{\sigma}\right\rangle\left\langle\hat{\boldsymbol{\epsilon}}_{\pi}^{\prime}|M| \hat{\boldsymbol{\epsilon}}_{\pi}\right\rangle
\end{array}\right),
$$

such that $\boldsymbol{V}^{\prime}=\mathbf{M} \cdot \boldsymbol{V}$. Consider the aforementioned coordinate transformation by an orthogonal matrix $R$ :

$$
\underbrace{R \cdot \boldsymbol{V}_{A}^{\prime}}_{\boldsymbol{V}_{B}^{\prime}}=\underbrace{R \cdot \mathbf{M}_{A} \cdot R^{-1}}_{\mathbf{M}_{B}} \cdot \underbrace{R \cdot \boldsymbol{V}_{A}}_{\boldsymbol{V}_{B}} .
$$

The same Jones matrix may be used with the coherency matrix formalism (section 1.4), so that the effect of a scattering process can also be studied for partially polarized beams. The density matrix of the scattered beam is then given by

$$
\rho^{\prime}=\mathbf{M} \cdot \boldsymbol{\rho} \cdot \mathbf{M}^{\dagger} .
$$

The intensity, $I^{\prime}$, and Poincaré-Stokes parameters, $\mathbf{P}^{\prime}$, of the scattered beam are easily obtained using Eqs. (15) and (16).

Subsequent diffraction off several optical elements, $\mathbf{M}_{1}, \mathbf{M}_{2}, \ldots$ (e.g., a monochromator, a phase plate, a sample, and a polarization analyzer) are represented by the product of the Jones matrices of the individual scatterers,

$$
\rho^{\prime}=\left(\ldots \mathbf{M}_{2} \cdot \mathbf{M}_{1}\right) \cdot \rho \cdot\left(\ldots \mathbf{M}_{2} \cdot \mathbf{M}_{1}\right)^{\dagger}=\ldots\left[\mathbf{M}_{2} \cdot\left(\mathbf{M}_{1} \cdot \rho \cdot \mathbf{M}_{1}^{\dagger}\right) \cdot \mathbf{M}_{2}^{\dagger}\right] \cdot \ldots
$$

The possible polarization sensitivity of a detector can be described by an additional Jones matrix acting as a polarization filter. Standard X-ray detectors, however, are not sensitive to the polarization of the detected radiation. Instead, polarization analyzers based on Bragg diffraction are used (see Sect. 2).

The Jones matrices for non-resonant magnetic scattering are given in [4], and those for resonant magnetic and ATS scattering have been tabulated in [5]. 


\subsection{Müller matrices}

The net effect of an ensemble of scatterers on the intensity and polarization can be elegantly described using the Müller calculus [11,12].

$$
S^{\prime}=\mathcal{M} \cdot S,
$$

where $\mathcal{M}$ is the Müller matrix, and $S_{i}=\operatorname{tr}\left(\boldsymbol{\sigma}_{i} \cdot \boldsymbol{\rho}\right)$ for $i=0,1,2,3$ is the Stokes vector with $\sigma_{0}=\left(\begin{array}{ll}1 & 0 \\ 0 & 1\end{array}\right)$ (see above).

The Müller matrix can be derived from the corresponding Jones matrix, $\mathbf{M}$ [22]:

$$
\mathcal{M}_{j, i}=\frac{1}{2} \operatorname{tr}\left(\boldsymbol{\sigma}_{i} \cdot \mathbf{M} \cdot \boldsymbol{\sigma}_{j} \cdot \mathbf{M}^{\dagger}\right) .
$$

The Jones matrix, however, contains the phase of the scattering process, therefore it cannot be determined from the Müller matrix which does not contain the phase information. The advantage of the Müller calculus is that it can also describe depolarizing elements. This is not possible with a single Jones matrix.

In the case of a large beam described by $\rho$ that diffracts of an ensemble of independent scatterers, e.g. different magnetic domains or grains of a mosaic crystal, the density matrix has to be calculated for each scatterer. Let $n$ enumerate the scatterers with probability $p(n)$, then $\boldsymbol{\rho}_{n}^{\prime}=\mathbf{M}_{n} \cdot \boldsymbol{\rho} \cdot \mathbf{M}_{n}^{\dagger}$. The composite scattered beam is then described by the sum of these density matrices, $\boldsymbol{\rho}^{\prime}=\sum_{n} p(n) \boldsymbol{\rho}_{n}^{\prime}$. Note that one does not obtain the same result by averaging over the individual Jones matrices!

The net effect of such an ensemble of scatters can be described by a single Müller matrix, $\mathcal{M}_{i j}=\frac{1}{2} \sum_{n} p(n) \operatorname{tr}\left(\boldsymbol{\sigma}_{i} \mathbf{M}_{n} \boldsymbol{\sigma}_{j} \mathbf{M}_{n}^{\dagger}\right)$, which is the average of the Müller matrices describing the individual scattering processes, $\mathcal{M}=\sum_{n} p(n) \mathcal{M}_{n}[22]$.

\section{Thomson scattering and linear polarization analyzer}

Thomson scattering is the elastic scattering by a free charged particle. It arises from the isotropic (scalar) polarizability of the scatterer, $f=f(\boldsymbol{Q}) \hat{\boldsymbol{\epsilon}}^{\prime \dagger} \cdot \hat{\boldsymbol{\epsilon}}$, where $f(\boldsymbol{Q})$ is the form factor, i.e. the Fourier transform of the particle's charge distribution. Thomson scattering can be observed in many classes of samples, such as gases, liquids, amorphous solids, single crystals or polycrystalline powders. In the context of resonant or anomalous scattering, Thomson scattering is referred to as "normal" or "charge" scattering. The corresponding Jones matrix is given by (using the coordinate system shown in Fig. 2)

$$
\mathbf{M}_{\mathrm{Th}}=\alpha\left(\begin{array}{ll}
1 & 0 \\
0 & \cos (2 \theta)
\end{array}\right),
$$

where $2 \theta$ is the scattering angle. The proportionality constant $\alpha$ contains all terms that are independent of the polarization, e.g. the form factor $f(\boldsymbol{Q})$ at the chosen scattering vector ${ }^{2} \boldsymbol{Q}=\boldsymbol{k}^{\prime}-\boldsymbol{k}$ in disordered systems or the structure factor $F(\boldsymbol{Q})$ in crystals, and geometrical term [24]. Note that Eq. (32) holds only in the kinematic approximation, i.e. in the absence of multiple scattering and anomalous effects.

\footnotetext{
${ }^{2}$ Both $\boldsymbol{Q}=\boldsymbol{k}^{\prime}-\boldsymbol{k}[5]$ and $\boldsymbol{Q}=\boldsymbol{k}-\boldsymbol{k}^{\prime}$ [4] are frequently used in the literature, the former predominantly in X-ray diffraction and the latter in neutron scattering. The sign of $\boldsymbol{Q}$ matters in non-centrosymmetric systems, e.g. for MAD-phasing of protein crystals [23]. When choosing one convention or the other, it is therefore important to also take into account the sign of the propagator (Eqs. (1) and (2)) and the definition of the Fourier transform used to calculate the structure factor.
} 
When an X-ray beam undergoes Thomson scattering, its Stokes parameters are modified as follows [25]:

$$
\begin{aligned}
\boldsymbol{\rho}^{\prime} & =\mathbf{M}_{\mathrm{Th}} \boldsymbol{\rho} \mathbf{M}_{\mathrm{Th}}^{\dagger}=\alpha\left(\begin{array}{cc}
1+P_{1} & \left(P_{2}-i P_{3}\right) x \\
\left(P_{2}+i P_{3}\right) x & \left(1-P_{1}\right) x^{2}
\end{array}\right) \\
I^{\prime} & =\frac{I}{2}|\alpha|^{2}\left[1+x^{2}+P_{1}\left(1-x^{2}\right)\right] \\
P_{1}^{\prime} & =\frac{1-x^{2}+P_{1}\left(1+x^{2}\right)}{1+x^{2}+P_{1}\left(1-x^{2}\right)} \\
P_{2}^{\prime} & =\frac{2 P_{2} x}{1+x^{2}+P_{1}\left(1-x^{2}\right)} \\
P_{3}^{\prime} & =\frac{2 P_{3} x}{1+x^{2}+P_{1}\left(1-x^{2}\right)},
\end{aligned}
$$

where $x=\cos (2 \theta)$.

For $\cos (2 \theta)=0, \mathbf{M}=\alpha\left(\begin{array}{ll}1 & 0 \\ 0 & 0\end{array}\right)$ (Eq. (32)), i.e. only the $\sigma$ component survives. The Stokes parameters (Eqs. (34)-(37)) of the scattered beam reduce to

$$
\begin{gathered}
I^{\prime}=\frac{I}{2}|\alpha|^{2}\left(1+P_{1}\right) \\
P_{1}^{\prime}=1 \\
P_{2,3}^{\prime}=0,
\end{gathered}
$$

except for the case $P_{1}=-1$, when the intensity of the scattered beam vanishes, $I^{\prime}=0$.

A Thomson scatterer with $2 \theta=90^{\circ}$, and that can be rotated by an angle $\eta$ about the beam axis, may thus be used as a linear polarization analyzer (PA) to determine the linear polarization parameters, $P_{1}$ and $P_{2}$ (see Fig. 4). Note that the degree of circular polarization, $P_{3}$, cannot be measured in this way. It is, however, possible to establish an upper bound, as $P_{3}^{2} \leq 1-P_{1}^{2}-P_{2}^{2}$.

Scatterers may be incoherent, e.g., an amorphous Kapton foil, or Bragg scatterers, in which case the condition $\cos (2 \theta)=0$ is generally fulfilled only approximatively, as a perfect match of the PA crystal's $d$-spacing to the desired photon energy cannot always be obtained (see table 1). In practice there is always some crosstalk between the polarization channels, $0<x \ll 1$, generally referred to as "spillover".

Typically only the intensity of the scattered beam is measured. It is given by (Eqs. (24), (34))

$$
I^{\prime} \propto S+P_{1} \cos (2 \eta)+P_{2} \sin (2 \eta),
$$

where $S=\left(1+x^{2}\right) /\left(1-x^{2}\right)=\left(1+\cos ^{2}(2 \theta)\right) /\left(\sin ^{2}(2 \theta)\right)$ is characteristic of the spillover; it is usually determined by measuring a beam of known polarization, e.g., the incident beam or a strong structural reflection of the sample.

In addition to the usual benefits of analyzer crystals, such as rejection of diffuse scattering and fluorescence as well as further reduction of higher harmonics of the $\mathrm{X}$-ray wavelength, PA offers further advantages which are specific to magnetic and resonant scattering. 


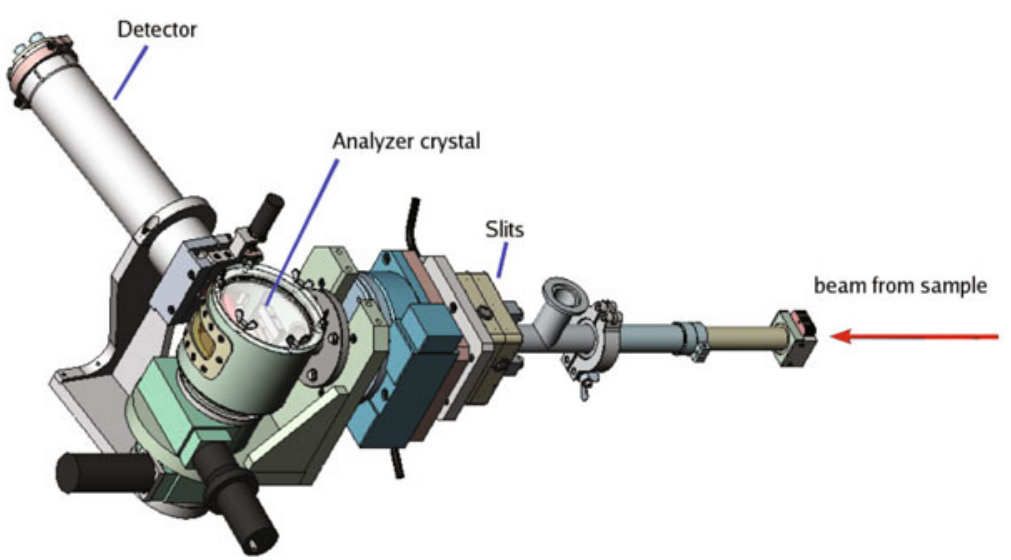

Fig. 4. Experimental configuration for linear polarization analysis of X-rays at the ESRF Beamline ID20 [26]. The beam scattered off the sample enters an evacuated flight tube (right). In-vacuum slits are positioned upstream of the analyzer crystal. The analyzer crystal and detector are mounted on a common goniometer rotating about the beam ( $\eta$-axis). The Bragg $\left(\theta_{\mathrm{PA}}\right)$ and scattering angles $\left(2 \theta_{\mathrm{PA}}\right)$ can be adjusted individually.

Table 1. A selection of crystals that can be used for polarization analysis. The photon energy at which $2 \theta_{\mathrm{PA}}=90^{\circ}$ is presented in column 3 , while columns 4,5 and 6 list some absorption edges for which the corresponding crystal represents a good choice.

\begin{tabular}{|c|c|c|c|c|c|c|}
\hline \multirow[t]{2}{*}{ Crystal } & \multirow[t]{2}{*}{ (H K L) } & \multirow{2}{*}{$\begin{array}{c}\mathrm{d} \\
{[\AA]}\end{array}$} & \multirow{2}{*}{$\begin{array}{c}E\left(90^{\circ}\right) \\
{[\mathrm{keV}]}\end{array}$} & \multicolumn{3}{|c|}{ Edges } \\
\hline & & & & $5 f \mathrm{M}_{4,5}$ & $4 f \mathrm{~L}_{1,2,3}$ & $3 d \mathrm{~K}$ \\
\hline $\mathrm{Au}$ & $\left(\begin{array}{lll}1 & 1 & 1\end{array}\right)$ & 2.355 & 3.72 & $\mathrm{U}, \mathrm{Np}$ & & \\
\hline $\mathrm{Pt}$ & $\left(\begin{array}{lll}1 & 1 & 1\end{array}\right)$ & 2.266 & 3.87 & & & \\
\hline $\mathrm{Cu}$ & $\left(\begin{array}{lll}2 & 0 & 0\end{array}\right)$ & 1.807 & 4.85 & & & $\mathrm{Ti}$ \\
\hline Graphite & $\left(\begin{array}{lll}0 & 0 & 4\end{array}\right)$ & 1.677 & 5.22 & & & $\mathrm{Ti}, \mathrm{V}$ \\
\hline Mo & $\left(\begin{array}{lll}2 & 0 & 0\end{array}\right)$ & 1.574 & 5.57 & & $\mathrm{La}, \mathrm{Ce}$ & $\mathrm{V}$ \\
\hline $\mathrm{Al}$ & $\left(\begin{array}{lll}2 & 2 & 0\end{array}\right)$ & 1.432 & 6.12 & & $\mathrm{La}, \mathrm{Ce}, \mathrm{Pr}, \mathrm{Nd}$ & $\mathrm{Cr}, \mathrm{Mn}$ \\
\hline $\mathrm{Cu}$ & $\left(\begin{array}{lll}2 & 2 & 0\end{array}\right)$ & 1.276 & 6.86 & & $\mathrm{Sm}, \mathrm{Nd}, \mathrm{Eu}$ & $\mathrm{Mn}, \mathrm{Fe}$ \\
\hline $\mathrm{Au}$ & $\left(\begin{array}{lll}2 & 2 & 2\end{array}\right)$ & 1.177 & 7.44 & & $\mathrm{Sm}, \mathrm{Eu}, \mathrm{Gd}$ & $\mathrm{Fe}, \mathrm{Co}$ \\
\hline $\mathrm{Al}$ & $\left(\begin{array}{lll}2 & 2 & 2\end{array}\right)$ & 1.169 & 7.49 & & $\mathrm{Sm}, \mathrm{Eu}, \mathrm{Gd}$ & $\mathrm{Fe}, \mathrm{Co}$ \\
\hline $\mathrm{Pt}$ & $\left(\begin{array}{lll}2 & 2 & 0\end{array}\right)$ & 1.133 & 7.74 & & $\mathrm{Sm}, \mathrm{Eu}, \mathrm{Gd}, \mathrm{Tb}, \mathrm{Dy}$ & $\mathrm{Co}$ \\
\hline Graphite & $\left(\begin{array}{lll}0 & 0 & 6\end{array}\right)$ & 1.118 & 7.84 & & $\mathrm{Eu}, \mathrm{Gd}, \mathrm{Tb}, \mathrm{Dy}, \mathrm{Ho}$ & $\mathrm{Co}$ \\
\hline $\mathrm{Cu}$ & $\left(\begin{array}{lll}2 & 2 & 2\end{array}\right)$ & 1.042 & 8.41 & & Gd, Tb, Dy, Ho, Er, Tm & $\mathrm{Ni}, \mathrm{Cu}$ \\
\hline $\mathrm{Pt}$ & $\left(\begin{array}{lll}4 & 0 & 0\end{array}\right)$ & 0.981 & 8.94 & & $\mathrm{~Tb}, \mathrm{Dy}, \mathrm{Ho}, \mathrm{Yb}$ & $\mathrm{Cu}$ \\
\hline $\mathrm{Pd}$ & $\left(\begin{array}{lll}4 & 0 & 0\end{array}\right)$ & 0.973 & 9.01 & & $\mathrm{~Tb}, \mathrm{Dy}, \mathrm{Ho}, \mathrm{Yb}$ & $\mathrm{Cu}$ \\
\hline Graphite & $\left(\begin{array}{lll}0 & 0 & 8\end{array}\right)$ & 0.839 & 10.48 & & $\mathrm{Tm}, \mathrm{Yb}, \mathrm{Lu}$ & $\mathrm{Zn}, \mathrm{Ga}$ \\
\hline $\mathrm{Au}$ & $\left(\begin{array}{lll}3 & 3 & 3\end{array}\right)$ & 0.785 & 11.16 & & & $\mathrm{Ge}$ \\
\hline
\end{tabular}

Magnetic X-ray scattering at synchrotron sources is mostly done in the vertical scattering geometry with incident $\sigma$ polarization. At dipole resonances, all magnetic scattering is the $\pi$ polarization channel. A polarization analyzer set to accept only the $\pi$ channel thus greatly improves the signal-to-noise ratio by preferentially suppressing Thomson scattering, which remains $\sigma$ polarized.

For practical applications some additional issues have to be considered. As was already pointed out, Eq. (32) neglects multiple scattering effects. Diffraction from near-perfect crystals where the dynamical theory of X-ray diffraction has to be applied 
will give rise to deviations from this behavior. In extreme cases, like the Renninger effect, the polarization dependence is totally different [2,27-29]. Furthermore, nearperfect crystals have very sharp rocking curves so that the instrumental resolution function is strongly modified upon rotating the PA about the scattered beam: In the $\sigma$ position, the acceptance will be narrow in the $2 \theta$ and wide in the $\chi$ directions of a normal 4-circle diffractometer [30], whereas in the $\pi$ position the opposite is true. For some experiments it is desirable to improve the longitudinal resolution. However, it is generally not convenient to work with varying resolution functions. In particular, measurements of the Poincaré-Stokes parameters, which require absolute values of the integrated intensity at several positions of the PA, have to be carried out with care.

In principle, it is possible to determine $P_{1}\left(P_{2}\right)$ from a pair of measurements at $\eta=0^{\circ}$ and $90^{\circ}\left(\eta= \pm 45^{\circ}\right) \quad[31,32]$. In order to estimate the systematic errors, however, we recommend a fit of Eq. (41) to measurements at different positions of $\eta$ between $0^{\circ}$ and $180^{\circ}$ in steps of $30^{\circ}$ or less. Furthermore, the intensities should be recorded by rocking the analyzer crystal rather than the sample [33]; in this way artifacts from the variable resolution function are minimized.

Finally, the sharp rocking curves require very high accuracy of the alignment, mechanical precision and software control during changes of the photon energy or the polarization channel. Therefore crystals with a moderate mosaic width on the order of $0.1^{\circ}$, for example metals, are preferred for most practical applications. In this case the resolution function is defined by the setting of slits before the PA assembly which do not rotate with the PA. A serious disadvantage of such crystals is their relatively low reflectivity, on the order of $1-10 \%$. The requirement of a fixed scattering angle $2 \theta_{\mathrm{PA}} \approx 90^{\circ}$ leads to a linear increase of the momentum transfer, $Q$, with increasing photon energy, and thus to diminishing form factors $f(Q)$. Thus, in general, the reflectivity of polarization analyzers decreases towards higher photon energies. A list of some selected PA crystals commonly used, together with the X-ray absorption edges they may be applied to, is presented in table 1 .

The selectivity of a polarization analyzer can be enhanced by reflecting the beam multiple times, e.g. using a channel-cut crystal. The throughput, i.e. the maximum transmitted intensity in the allowed polarization channel, however, will drop drastically unless perfect crystals such as Si or Ge are used.

Recently, a polarization purity of $2 \cdot 10^{-9}$ was achieved using channel-cut Si crystals with 4 reflections [34] with a photon energy tuning to fulfill the $2 \theta=90^{\circ}$ condition as close as possible. The polarization purity in that experiment was limited by multiple-beam diffraction effects (Renninger effect) that partially rotate the plane of polarization and thus contaminate the "forbidden" channel.

\section{Polarization analysis of resonant scattering at the Ge K-edge}

As an example of polarization analysis we present the K-edge resonant scattering at the (006) reflection of $\mathrm{Ge}[33,35-38]$. This reflection is crystallographically forbidden due to a glide plane extinction rule., i.e. the structure factor $F(\boldsymbol{Q})$ vanishes for scalar (Thomson) scattering. Rank-3 anisotropic tensor scattering (ATS), however, is allowed [35]. Due to the high symmetry of Ge, the ATS cross section is completely determined by symmetry, up to a (resonant) amplitude factor that varies as the photon energy is tuned across the resonance. At each given photon energy $\hbar \omega$ the polarization of the scattered beam as function of the the azimuth, $\psi$ (i.e. upon rotation of the crystal about the scattering vector $\boldsymbol{Q}[24])$ is completely free of adjustable parameters. For incident $\sigma$ polarization the Poincaré-Stokes parameters of the scattered 

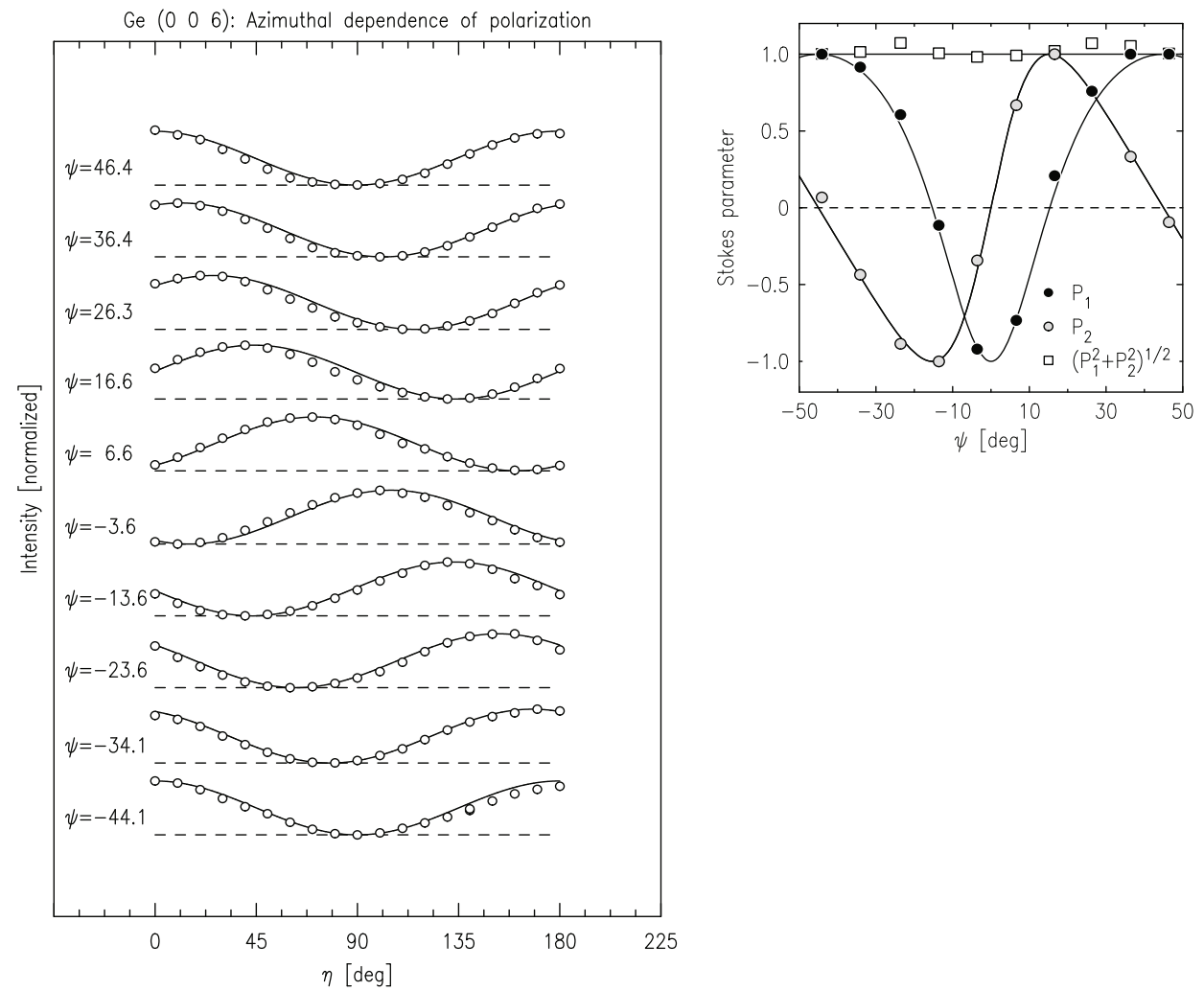

Fig. 5. Left: measurement of the Poincaré-Stokes parameters as function of the azimuthal angle at the Ge (006) reflection. Solid lines are fits to Eq. (41). Right: Poincaré-Stokes parameters extracted from the measurements shown on the left by fitting to Eq. (41). The experimental values are in quite satisfactory agreement with the parameter-free predictions (Eqs. (42) and (43)), show as lines. Taken from [33].

beam are given by $[33,37,38]$,

$$
\begin{aligned}
& P_{1}(\theta, \psi)=\frac{\sin ^{2}(2 \psi)-\sin ^{2}(\theta) \cos ^{2}(2 \psi)}{\sin ^{2}(2 \psi)+\sin ^{2}(\theta) \cos ^{2}(2 \psi)} \\
& P_{2}(\theta, \psi)=\frac{\sin (4 \psi) \sin (\theta)}{\sin ^{2}(2 \psi)+\sin ^{2}(\theta) \cos ^{2}(2 \psi)} \\
& P_{3}(\theta, \psi)=0,
\end{aligned}
$$

where $\theta$ is the Bragg angle. In particular, we note that $P_{1}^{2}+P_{2}^{2}=1$ for all azimuths, i.e. the scattered beam is always fully linearly polarized.

Experimental data were taken as follows [33]: The photon energy was tuned to the maximum of the resonance, $11.096 \mathrm{keV}$. For each setting of the azimuth, the Ge (006) reflection was aligned. Then the polarization analyzer was rotated about the beam in steps of $10^{\circ}$. At each position the integrated intensity was recorded by rocking the $\mathrm{Au}(333)$ analyzer crystal. These integrated intensities were then fit to Eq. (41) in order to extract the Poincaré-Stokes parameters, see Fig. 5 (left). The final results are shown in Fig. 5 (right), along with the predictions of Eqs. (42) and (43). The 
agreement between the experimental data and the theoretical predictions is quite satisfactory.

Unfortunately, the polarization dependence is identical for the two possible microscopic mechanisms of the resonance, an E1-E2 mixed resonance $[35,37,38]$, or a E1 resonance of atoms displaced from their equilibrium positions by thermal motion (thermal motion induced scattering, TMI) [36]. Therefore this experiment was unable to distinguish between the two competing models. Studies of the amplitude and line shape as function of temperature, however, favor the TMI model in Ge [36,39], whereas in $\mathrm{ZnO}$ both modes are significant [40].

\section{Complete characterization of the polarization state}

The complete determination of the polarization state of a synchrotron beam is essential for characterizing the circular polarized light produced by using X-ray phase plates or exotic insertion devices. Two main methods have been successfully used for this purpose.

The first is based on the same principle as used in the visible region: A full characterization of the polarization of a wave (in terms of the Poincaré-Stokes vector) can be obtained by using the combination of a phase retarder and a linear polarization analyzer [11]. In the X-ray regime, this was first proposed by [41] and then used by [42].

The second polarimetry method is to use multiple beam diffraction. The phase probing capability of this method can allow for the unambiguous determination of the Poincaré-Stokes parameters and can be applied to X-ray polarimetry $[28,43]$.

The authors thank F. de Bergevin, S.D. Brown, V. Dmitrienko, T. Roth, and S.B. Wilkins for many stimulating discussions. The ESRF is acknowledged for provision of beam time on beamline ID20.

\section{References}

1. M. Hart, Phil. Mag. B 38, 41 (1978)

2. V.A. Belyakov, V.E. Dmitrienko, Sov. Phys. Usp. 32, 697 (1989)

3. F. de Bergevin, M. Brunel, Phys. Lett. A 39, 141 (1972)

4. M. Blume, D. Gibbs, Phys. Rev. B 37, 1779 (1988)

5. J.P. Hill, D.F. McMorrow, Acta Crystallogr. A 52, 236 (1996)

6. J.P. Hannon, G.T. Trammell, M. Blume, D. Gibbs, Phys. Rev. Lett. 61, 1245 (1988)

7. P. Carra, B.T. Thole, M. Altarelli, X. Wang, Phys. Rev. Lett. 70, 694 (1993)

8. S.W. Lovesey, S.P. Collins, X-ray scattering and absorption by magnetic materials (Oxford University Press, 1996)

9. S. Di Matteo, Y. Joly, A. Bombardi, L. Paolasini, F. de Bergevin, C.R. Natoli, Phys. Rev. Lett. 91, 257402 (2003)

10. C.G. Barkla, Proc. Roy. Soc. 77, 247 (1906)

11. M. Born, E. Wolf, Principles of Optics, 7th edn. (Cambridge University Press, 1999)

12. D.H. Goldstein, Polarized Light, 3rd edn. (CRC Press, 2011)

13. G.G. Stokes, Trans. Cambr. Phil. Soc. 9, 399 (1852)

14. F. de Bergevin, M. Brunel, Acta Crystallogr. A 37, 314 (1981)

15. S.W. Lovesey, S.P. Collins, J. Synchrotron Rad. 8, 1065 (2001)

16. F.W. Lipps, H.A. Tolhoek, Physica 20, 85 (1954)

17. U. Fano, Rev. Mod. Phys. 29, 74 (1957)

18. F.W. Lipps, H.A. Tolhoek, Physica 20, 395 (1954)

19. R.C. Jones, J. Opt. Soc. Am. 31, 448 (1941)

20. H. Hurwitz, Jr, R.C. Jones, J. Opt. Soc. Am. 31, 493 (1941) 
21. R.C. Jones, J. Opt. Soc. Am. 31, 500 (1941)

22. K. Kim, L. Mandel, E. Wolf, J. Opt. Soc. Am. A 4, 433 (1987)

23. J.L. Hodeau, V. Favre-Nicolin, S. Bos, H. Renevier, E. Lorenzo, J.F. Berar, Chemical Reviews 101(6), 1843 (2001), http://pubs.acs.org/doi/pdf/10.1021/cr0000269, http://pubs.acs.org/doi/abs/10.1021/cr0000269

24. B.E. Warren, X-ray diffraction (Dover Publications, Inc., 1990)

25. S. Ramaseshan, G.N. Ramachandran, Acta Cryst. 6, 364 (1953)

26. L. Paolasini, C. Detlefs, C. Mazzoli, S. Wilkins, P.P. Deen, A. Bombardi, F. de Bergevin, F. Yakhou, J.P. Valade, I. Beslavetz, et al., J. Synchrotron Rad. 14, 301 (2007)

27. Q. Shen, K.D. Finkelstein, Phys. Rev. Lett. 65, 3337 (1990)

28. Q. Shen, K.D. Finkelstein, Phys. Rev. B 45, 5075 (1992)

29. Y.P. Stetsko, H.J. Juretschke, Y.S. Huang, C.H. Chao, C.K. Chen, S.L. Chang, Acta Crystallogr. 56, 394 (2000)

30. W.R. Busing, H.A. Levy, Acta Cryst. 22, 457 (1967)

31. F. Vaillant, Acta Cryst. A 33, 967 (1977)

32. J.A. Paixão, C. Detlefs, M.J. Longfield, R. Caciuffo, P. Santini, N. Bernhoeft, J. Rebizant, G.H. Lander, Phys. Rev. Lett. 89, 187202 (2002)

33. C. Detlefs, Physica B 345, 45 (2004)

34. B. Marx, I. Uschmann, S. Höfer, R. Lötzsch, O. Wehrhan, E. Förster, M. Kaluza, G.G. Paulus, T. Stöhlker, H. Gies, et al., Optics Comm. 284, 915 (2011)

35. D.H. Templeton, L.K. Templeton, Phys. Rev. B 49, 14850 (1994)

36. J. Kokubun, M. Kanazawa, K. Ishida, V.E. Dmitrienko, Phys. Rev. B 64, 73203 (2001)

37. I.S. Elfimov, N.A. Skorikov, V.I. Anisimov, G.A. Sawatzky, Phys. Rev. Lett. 88, 15504 (2002)

38. I.S. Elfimov, N.A. Skorikov, V.I. Anisimov, G.A. Sawatzky, Phys. Rev. Lett. 88, 239904(E) (2002)

39. A.P. Oreshko, V.E. Dmitrienko, E.N. Ovchinnikova, J. Math. Sci. 172, 859 (2011)

40. S.P. Collins, D. Laundy, V.E. Dmitrienko, D. Mannix, P. Thompson, Phys. Rev. B 68, $064110(2003)$

41. T. Ishikawa, K. Hirano, S. Kikuta, J. Appl. Cryst. 24, 982 (1991)

42. C. Giles, C. Vettier, F. de Bergevin, C. Malgrange, G. Grübel, F. Gossl, Rev. Sci. Instrum. 66, 1518 (1995)

43. Q. Shen, K.D. Finkelstein, Rev. Sci. Instrum. 64, 3451 (1993) 